\title{
Essai sur la psychologie de la canine
}

\section{RÉSUMÉ}

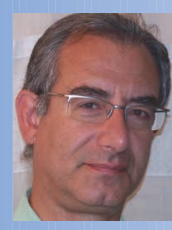

Marc Gérald CHOUKROUN

Docteur en chirurgie dentaire Paris VII, Spécialiste qualifié

en orthopédie dento-faciale

(CECSMO) Paris V,

CES de biologie buccale /

Odontologie légale / ODF,

Maîtrise de psychologie Paris VII Sorbonne,

51, avenue Henri Ginoux,

92120 M ontrouge.

La canine est affectée d'une symbolique particulière. Assimilée à «une dent de chien», le public la pense volontiers longue et pointue, faite pour percer la peau et provoquer le saignement. Le mythe qui regroupe tous ces objets métaphoriques est le mythe du vampire, que nous nous proposons d'étudier dans cet article.

Nous envisagerons les origines du mythe, les hypothèses médicales, les sources culturelles, puis nous tenterons de discuter les interprétations psychanalytiques. Pouvons-nous en tirer des informations utiles en clinique ? Ce sera le mérite de cette étude d'y aboutir. Nous ne pouvons citer d'auteurs précis, notre recherche a été effectuée sur le WEB.

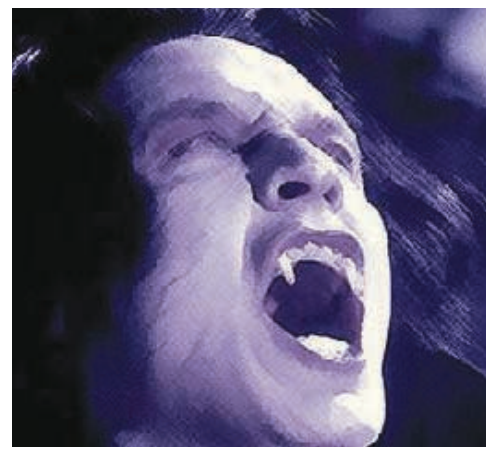

- canine

- symboles

- psychologie

- vampire 


\section{Les origines du mythe}

$>$

Nous apprenons, que le mythe du vampire se fonde sur une très ancienne mythologie du cannibalisme. Mythologie qui se retrouve dans de nombreuses civilisations. Cependant plus spécifiquement, et différemment de la recherche de l'absorption du corps de l'autre, c'est le sang de l'autre qui est perçu comme liquide symbolisant la vie. En buvant le sang de l'autre, la fin visée est de prendre la vie de l'autre. On retrouve un vase préhistorique en Perse où un monstre suce le sang d'un homme. Des légendes se retrouvent aussi bien chez les chinois, les aztèques, les indiens, les malais, les esquimaux, etc.

Plus proche de nous, il faut citer le christianisme comme une révolution, car c'est le Christ luimême qui invite à être incorporé par les fidèles : «ceci est mon sang, ceci est mon corps».
On comprendra que la sémantique sousjacente à cette mythologie est liée au désir d'immortalité : prendre la vie pour prolonger la sienne. Prendre le corps du Christ pour accéder auprès de Dieu.

L'apparition au $18^{e}$ siècle du mythe de Dracula, fait référence au retour du "gothique» : la cape, le château isolé, l'aspect tyrannique, la terreur paysanne. II représente une rébellion contre la religion chrétienne en brandissant un imaginaire hérétique. En outre, on reconnaîtra une symbolique sado-masochiste issue du libertinage et de la pensée de Sade. II va se constituer en un mythe et traversera le $19 \mathrm{e}$, le $20^{\mathrm{e}}$ siècle, jusqu'à nos jours. Il contient donc les éléments cruciaux de notre civilisation moderne.

\section{L'histoire de Dracula}

Le premier texte faisant référence aux vampires est de John Stagg en 1810 («the Vampyre»); puis c'est le roman de John William Pilidori en 1819 («le Vampire»); puis le thème devient une mode (Théophile Gautier, Hoffman, Tolstoï, ...).

C'est un professeur de langues orientales de Budapest qui raconte à Bram Stocker, un auteur irlandais, la légende de Vlad Tepes (l'empaleur en roumain), prince sanguinaire de Valachie et de Transylvanie puis des Carpates au $15^{\mathrm{e}}$ siècle. II reprend le surnom de son père Dracula de Drac : «le diable».

Bram Stocker crée alors le comte de Dracula et le roman paraît en 1897.
En 1924, Dracula est adapté au théâtre de Londres par Hamilton Dean. La pièce a un tel succès qu'elle sera reprise à Broadway.

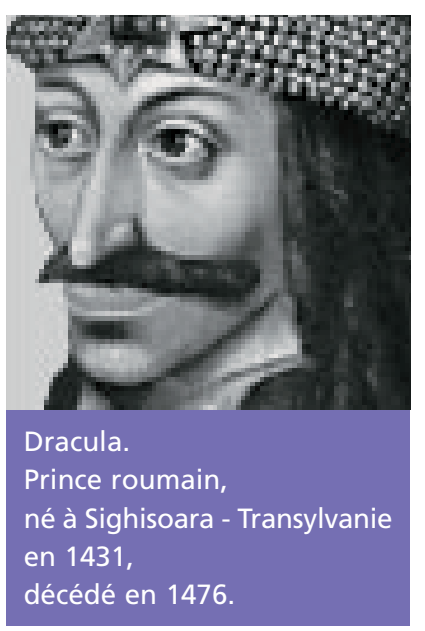


En 1922, un film est édité : «Nosferatu le Vampire» de Friedrich Wilhelm Murnau. Ce sera le début d'une longue série de films sur

\section{L’aspect médical}

Le besoin de récupérer du sang pour améliorer sa santé n'est pas execptionnel. On conseille couramment aux convalescents de manger de la viande saignante.

Mais le personnage le plus célèbre est sans doute la comtesse Erzebet Bathory du 16e siècle qui, dans son château des Carpates, saignait des centaines de jeunes filles pour se baigner dans leur sang et le boire.

Dans les années cinquante en Angleterre, John Haigh, surnommé le vampire de Londres assassina neuf personnes pour boire leur sang.

Cette pathologie nommée «hématomanie», semble répertoriée et connue aux USA (50 000 cas). Ces personnes reçoivent du sang de le thème des vampires. La dernière version célèbre est celle de Francis Coppola en 1992.

volontaires, pour obtenir des effets surnaturels. Ils se regrouperaient même en réseaux. Cette pathologie serait liée à une enfance douloureuse et présente des photophobies.

Un certain Dr Dolphin, ayant bien étudié la symptomatologie du vampire, associe ces observations à une maladie des facteurs sanguins : la porphyrie.

Selon cet auteur, la porphyrie présente des malformations dentaires, notamment des canines pointues, une intolérance à la lumière, et donc une peau très blanche. Une substance contenue dans l'ail accentuerait la maladie. Elle serait fréquente dans certaines régions reculées des Carpates.

\section{Linterprétation psychanalytique}

Freud décrit les perversions comme des déviations sexuelles de deux types:

- déviation quant à l'objet: le partenaire sexuel n'est pas celui attendu par le couple normal (invertis, pédophilie, zoophilie, etc.) ;

- déviation quant au but: le rapport génital n'est pas respecté (fétichisme, sado-masochisme, exhibitionnisme, etc.).

Le vampirisme qui présente une sexualité dont le but est la morsure au cou du partenaire, appartient donc aux déviations sexuelles.
Nous l'avons dit, elle vise une immortalité en prenant la vie de l'autre. Or Platon dans le «banquet» explique que la finalité du désir est de produire un enfant. Schopenhauer quant à lui, explique que la reproduction répond à une immortalité de l'espèce.

Ainsi le vampirisme serait un désir d'immortalité égocentriste.

Platon pose également qu'il existe deux catégories d'Eros: I'Aphrodite uranienne ou céleste et l'Aphrodite vulgaire. L'amour céleste est vertueux et se déclare, tandis que I'amour vulgaire se cache. 
L'homme moderne projette dans le vampirisme, les pulsions sexuelles infantiles et hostiles. Rejetées par l'adulte et la société, ces pulsions refoulées font retour dans l'expression artistique.

En se levant la nuit, le vampire rappelle que c'est à travers nos rêves que s'expriment ces pulsions.
La canine, par sa forme animale et phallique, représente métaphoriquement cette sexualité interdite.

L'extinction du vampire ne se fait-elle pas d'ailleurs par la mise d'un pieu dans le cœur, c'est-à-dire par la victoire d'un objet phallique vertueux ?

\section{Conséquences cliniques}

Dans la relation transférentielle, praticienpatient, le patient infantilisé par sa crainte ou sa douleur, régresse et projette parfois sur le praticien ses propres pulsions. II dénoncera alors ce dernier comme sadique, ou investi de pulsions hostiles.

En sommes dans le duo clinique, le patient s'imagine comme victime innocente et prend le praticien pour un vampire (voire assoiffé de sang, dans l'acte chirurgical).

Face à ce comportement, le praticien peut se sentir agressé et produire du contre-transfert. Sa réaction consiste à se justifier sur ses actes cliniques (culpabilité) ou à prendre une position d'indifférence (mise à distance de l'agresseur).

Une telle situation peut-être préjudiciable aux soins; soit par une conversation prolongée et laissant le patient insatisfait, soit par un manque d'informations et de ce fait, un non respect du consentement éclairé.

Ces mécanismes de projection phobiques trouveront une meilleure résolution dans l'écoute. Le patient, alors libéré de ses pulsions, sera à même de suivre une consultation dans de bonnes conditions et surtout de laisser le praticien occuper son corps.
Exemple clinique :

Le patient: - Qu'est-ce que vous allez me faire?

Le praticien :- Une petite injection.

Le patient : - Vous allez me faire mal !

Le praticien : - Non c'est une aiguille très fine.

Le patient : - C'est-ce que vous dites... Et où allez-vous l'injecter?

Le praticien :- Dans la gencive, vous ne sentirez rien.

Le patient : - La dernière fois on $\mathrm{m}^{\prime} \mathrm{a}$ dit cela et j'ai eu mal pendant une semaine !

Le praticien : - Il y avait peut-être une infection, ou un petit vaisseau ?

Le patient :- Oui il y a toujours quelque chose après et rien avant !

Le praticien: - Écoutez, nous perdons du temps, il faut que je vous endorme...

Le patient: - C'est cela, endormez-moi pour que je puisse rien dire !

Le praticien :- On peut ne rien faire aussi ! Le patient : - Je ne suis pas tout à fait prêt...

Ainsi le dialogue peut durer jusqu'à ce que I'une des parties renonce...

Reprenons cette situation avec un praticien qui aurait compris que le patient produit des fan- 
tasmes pour exprimer ses pulsions et qui est à l'écoute.

Le patient: - qu'est-ce que vous allez me faire ?

Le praticien : - Je vous sens mal à l'aise, que se passe-t-il ?

Le patient : - J'ai peur...

Le praticien :- Oui de quoi ?

Le patient: - La dernière fois, cela s'est mal passé, j'ai eu mal toute la semaine.

Le praticien :- De quoi avez-vous peur ?

Le patient : - De mourir...

\section{Conclusion}

Le mythe du vampire, en tant qu'il cache une sexualité interdite, mais qui appartient au monde fantasmagorique de chacun, est bien un mythe de notre temps. L'odonto-stomatologiste n'y échappe pas et à son insu !

L'écoute du patient est essentielle, à condition de savoir ce qu'il faut écouter. Alors, le patient se sent compris et la réponse du praticien peut être accompagnante. Cette démarche change
Le praticien : - Pourquoi ?

Le patient : - Parce que j'aime la vie.

Le praticien: - Vous avez raison, moi aussi, c'est pourquoi je vais bien vous soigner.

Le patient : - Ah merci... je préfère cette idée docteur!

Ainsi cet exemple montre qu'en laissant la peur s'exprimer, le patient verbalise très vite son fantasme. La peur de mourir n'est qu'une image, qui rappelle le mythe d'immortalité du vampire... Agression, peur, immortalité : tout est dit. Et le patient termine sa régression pour se prendre en charge.

radicalement l'aspect clinique. Le patient s'apaise et donne son consentement. Le praticien, loin d'être agressé, est reconnu dans son soutien charismatique.

Quant à la morphologie de la canine vampirique, elle signe la plupart du temps une supraclusion sévère et mérite d'être traitée orthodontiquement!

\section{Références}

WEB :

keys words :

"vampire", "Dracula".
Choukroun Marc Gérald.

"Au cœur de la relation

thérapeutique».
Glyphe édit, Paris, 2007. 


\section{SUMMARY}

\section{About the psychology of the canine}

Marc Gérald CHOUKROUN

Keywords

- canine

- symbole

- psychology

- vampire
The term Canine was formed from the tooth of a dog. It is commonly admitted that they hurt the skin providing blud. All the métaphoris concerned are assembled through the myth of the vampire. It will be the purpose of this article to explore this myhtology.

As a matter of fact we are supposed to discover the origins of the myth, its médical signification, its cultural sources and we will discuss the psychanalitycal interpretations.

Is it usefull for the clinician? We will try to prove it.

Our bibliography is light, the reason why, that our research was made through out the web... 